\title{
Knowledge, attitudes and practices on schistosomiasis and soil-transmitted helminths among caregivers in Ingwavuma area in uMkhanyakude district, South Africa
}

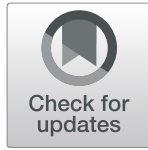

Hengiwe Sacolo-Gwebu* (D), Muhubiri Kabuyaya and Moses Chimbari

\begin{abstract}
Background: Schistosomiasis and soil-transmitted helminth infections are among the most chronic infections worldwide. Based on their demonstrable impact on human health, the WHO recently recommended the implementation of robust strategies aimed at controlling or eliminating schistosomiasis and soil-transmitted helminths by 2020. The implementation of this strategy, however, warrants a clear understanding of the community's knowledge, attitudes and practices in relation to these infections. This study sought to identify sociocultural gaps that should be addressed to ensure the success of cost-effective community-based schistosomiasis-soil-transmitted helminths control and elimination programs.
\end{abstract}

Methods: This was a cross-sectional mixed methodology study. Quantitative data were collected using a structured questionnaire from 442 caregivers of preschool aged children. In-depth interviews and focus group discussions were conducted among caregivers, preschool teachers, traditional authorities and community caregivers. All interviews were captured using an audio recorder to maximize accuracy. Quantitative data were analysed using bivariate and multivariate techniques while qualitative data were analysed thematically.

Results: Findings reflected inadequate knowledge, attitudes and practices in relation to schistosomiasis and soiltransmitted helminths while awareness of schistosomiasis and soil-transmitted helminths was high (87.1 and 79.2\% respectively). Correct knowledge on transmission, prevention, signs and symptoms and life cycle was low (below 50\%) for both infections among those who had heard of the disease. From multivariate analysis, being aged at least 35 years increased the odds of reporting good practices on schistosomiasis by $65 \%$ (COR 1.652, 95\% Cl: 1.073-2.543) while receiving health information through community meetings (COR 0.072, 95\% Cl: $0.010-0.548$ ) significantly reduced the odds of having good knowledge on schistosomiasis.

Conclusions: These findings are valuable in designing behavioural change approaches towards enhancing health outcomes through community-based interventions to ensure effective control and elimination of schistosomiasis and soil-transmitted helminths. There is a critical need for channelling efforts towards making health education the core of schistosomiasis and soil-transmitted helminths programs aimed at achieving intensified control or elimination of these infections by 2020.

Keywords: Knowledge, Attitudes, Practices, Schistosomiasis, Soil-transmitted helminths, Caregivers, Community members, uMkhanyakude district, South Africa

\footnotetext{
* Correspondence: hnsacolo@gmail.com

School of Nursing and Public Health, College of Health Sciences, Howard

College Campus, University of KwaZulu-Natal, Durban, South Africa
}

(c) The Author(s). 2019 Open Access This article is distributed under the terms of the Creative Commons Attribution 4.0 International License (http://creativecommons.org/licenses/by/4.0/), which permits unrestricted use, distribution, and reproduction in any medium, provided you give appropriate credit to the original author(s) and the source, provide a link to the Creative Commons license, and indicate if changes were made. The Creative Commons Public Domain Dedication waiver (http://creativecommons.org/publicdomain/zero/1.0/) applies to the data made available in this article, unless otherwise stated. 


\section{Background}

Schistosomiasis and soil-transmitted helminths (STH) are the most prevalent neglected tropical diseases (NTD) among disadvantaged communities in rural areas [1]. Sub-Saharan Africa (SSA) carries the heaviest burden of schistosomiasis and STH estimated at $90 \%$ of the global prevalence [2]. Schistosomiasis affects at least 200 million people [3] whilst STH affects more than a billion people globally [4]. Although schistosomiasis and STHs share significant similarities $[5,6]$, their life cycles and treatment strategies differ. For instance, schistosomiasis requires an intermediate host snail to complete its cycle and praziquantel for treatment whilst STHs complete their life cycle in the soil and Albendazole for treatment. Schistosomiasis and STH are associated with negative acute and chronic physical and cognitive outcomes. The long-term impact of these infections may cause permanent disability or even death in some cases.

Schistosomiasis and STH can be controlled using three key approaches which include improved sanitation, mass drug administration and health education [7, 8]. Other interventions for infection prevention include access to safe water, basic sanitation, and the promotion of hygiene practices [9-12]. These interventions play a critical role in the prevention and successful control or elimination of schistosomiasis and STH. Due to the commonalities between schistosomiasis and STH, their modes of prevention and transmission are often confused by community members and school aged children (SAC). The integration of schistosomiasis and STH control programs is therefore important to ensure that clear health education messages are crafted and disseminated. The success of community-based control initiatives depends on community acceptance of the programme [10, 13]. This implies that programs should first seek to understand the community's knowledge, attitudes and practices (KAP) towards schistosomiasis and STH before implementing program activities. Among community members, caregivers (parents and guardians) are considered a critical group due to their functional role as caregivers. Caregivers are highly influential within their families and at the community level, however without enough knowledge, they tend to mislead others or engage in risky practices that predispose their young children to schistosomiasis and STH infections [14, 15].

In rural areas of KwaZulu-Natal in South Africa, where schistosomiasis [16, 17] and STHs [18] are endemic, community-based KAP studies on schistosomiasis and STH are limited. This negatively impacts the effectiveness and sustainability of the control interventions aiming at eliminating schistosomiasis and STH at the community level [19]. According to recent findings, caregivers are critical in the control of schistosomiasis and STH among preschool aged children (PSAC) [15].
Studies conducted in Nigeria reported that caregivers predispose their children to schistosomiasis through risky water-related practices [20]. There is however lack of detailed understanding of the underlying reasons for such behaviour among caregivers. A study that reviewed KAP on schistosomiasis in sub-Saharan Africa (SSA) indicated the importance of including caregivers in the planning and implementation of control programmes for enhanced efficiency among PSAC [15]. The aim of this study, therefore, was to investigate the knowledge, attitudes and practices on schistosomiasis and soil-transmitted diseases among community members of the Ingwavuma area in uMkhanyakude District, South Africa.

\section{Methods}

Study area

The Ingwavuma area is in the North-Western part of uMkhanyakude District in KwaZulu-Natal, South Africa. It is arid and sub-tropical marked by erratic rainy and dry seasons. It also characterised by a hot and humid summer from November to February and a cooler, drier winter from June to August [21]. The hydrological network is formed by the Pongola and Ingwavuma rivers. Most communities in the Ingwavuma have limited access to piped water and as such depend on rivers, dams and ponds to sustain their domestic water needs [22].

\section{Study design and sampling Questionnaire survey}

We conducted a cross-sectional study among caregivers who were present in preschools and ECD centres during the screening of their PSAC under their care for schistosomiasis and STH. Participants belonged to villages located in the north-western part of that Ingwavuma area that was purposely selected into the study based on previous reports of schistosomiasis [22] and STH [23] among SAC in the area. The sample size for this study was linked to the sample size determined for the PSAC screening through the Cochran formula [24] for populations with unknown disease prevalence to yield a representative sample for larger populations. The minimum sample size required size through Cochran's formula calculation was 398. However, all caregivers present on the day of PSAC screening in ECD centres and preschools were recruited into the study (Fig. 1).

\section{Key informant in-depth interviews}

In-depth interviews were conducted among 62 key informants (traditional authorities, preschool teachers, ECD practitioners, community caregivers (CCGs) and nurses within the Ingwavuma area. The KAP key informant interview guide was developed, pre-tested, amended accordingly and used to interview the 


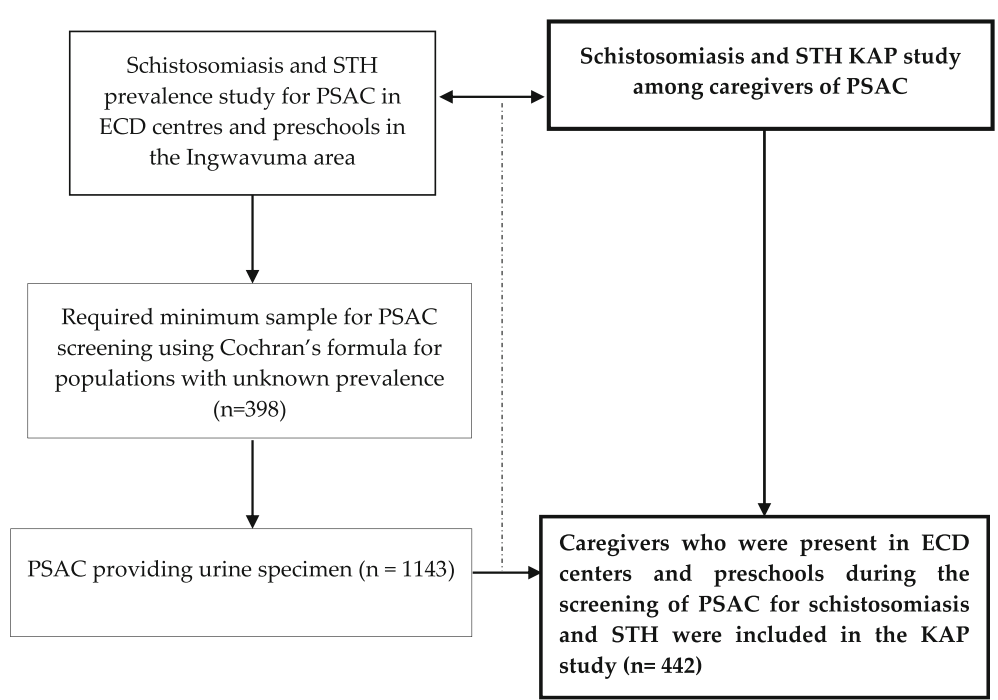

Fig. 1 KAP survey sampling framework

selected participants on various KAP aspects relating to schistosomiasis and $\mathrm{STH}$.

\section{Focus group discussions}

There were 138 participants who participated in 11 FGDs, averaging to at least 10 participants per FGD. The size of FGDs was within the range of the recommended size of 8 to 12 participants for FGDs. We conducted focus group discussions (FGDs) categorized by gender among caregivers of PSAC (8 FGDs) and CCGs (3 FGDs). The FGDs were conducted and led by trained moderators and note-takers fluent in isiZulu, the local language. Participants were purposively selected based on similar characteristics. Data collected were analysed thematically. Emerging themes and sub-themes were used to probe community members, whilst those taking notes focused on capturing non-verbal information and expressions that were evident. At the end of each interview, the research team held a short meeting to validate the collected information. FGDs were conducted in four main villages, purposely picked due to prior reported high prevalence among school aged children within our study area $[22,25,26]$. In each selected village, two FGD discussions were conducted comprising of ten members each as recommended by previous authors [27]. FGDs were grouped by gender nature of a rural setting where females are expected to be reserved in the presence of men.

\section{Data collection and management}

Quantitative data was collected using KoBo Collect software [28] and subsequently imported into SPSS. Statistical analysis was done after data validation.
Qualitative data from interviews was transcribed per verbatim and typed into an excel spreadsheet. A code sheet was developed for the focus group discussions after which, the data was coded into selected themes and was analyzed. Researchers checked for consistency of the transcripts against the audio files to ensure the accuracy of the transcribed files.

\section{Outcome variables}

The study assessed knowledge, attitudes and practices on STH and schistosomiasis. Firstly, total scores were determined by aggregating scores from responses to questions on knowledge and practices. Average scores were then calculated by dividing the total score by the number of questions in the respective attribute. Knowledge was defined as low or high level for scores below and above average. Similarly, practices were defined as poor or good practices for scores below and above the average score. We conducted a reliability test on the tool that assessed attitudes on schistosomiasis and $\mathrm{STH}$ comprising of14 items. Using Cronbach's alpha, the tool achieved a reliability score of $\alpha=0.704$ and was deemed reliable. All items appeared to decrease the alpha when deleted from the selection and were, therefore, retained for the study.

\section{Data analysis}

Chi-square test was used to assess associations between variables. A $p$-value of $<0.05$ was considered statistically significant. Multivariate analysis was conducted to assess the effect of background characteristics on practices and knowledge. Significant variables from the chi-square test were used as predictors in the multivariate analysis on 
practices and knowledge levels for both schistosomiasis and STH. Results from the binary logistic regression were expressed as crude odds ratios (COR) and adjusted odds ratio (AOR) with their 95\% confidence intervals (CI). The qualitative data were analyzed using content and thematic analysis to identify emerging themes. The process of analysis involved acquaintance with the data and development of codes based on our research findings.

\section{Results}

\section{Sociodemographic characteristics of PSAC caregivers}

Table 1 presents the overall sample characteristics. A total of 442 caregivers comprising of 28 (6.3\%) males and 414 (93.7\%) females participated in the study. The participants were caregivers to 442 PSAC that had been screened for schistosomiasis and STH. The majority (63.1\%) of the caregivers were in the 15-34 year age group while $364(82.4 \%)$ of the caregivers were unmarried.
Socio-demographic characteristics of participants who took part in FGDs and in-depth interviews

We conducted 3 FGDs among CCGs and 8 FGDs among caregivers on KAP in relation to schistosomiasis and STH. A total of 62 CCGs, preschool teachers and traditional authorities responded to schistosomiasis and STH KAP interviews. There was a single male respondent among CCGs and a single female respondent among traditional authorities. All preschool teachers were females and the youngest respondent in in-depth interviews was 35 years old. More than two-thirds of CCGs were born in the study area.

Schistosomiasis awareness, knowledge of signs, symptoms, transmission, life cycle and prevention among caregivers

Most study participants (87.1\%) acknowledged having previously heard of schistosomiasis (Fig. 2). Out of these, almost two-thirds knew signs and symptoms (69.1\%) and prevention measures $(61.6 \%)$ of schistosomiasis while half $(48.3 \%)$ and only $5 \%$ knew about treatment and control and life cycle respectively. These notable

Table 1 Caregivers' knowledge of schistosomiasis and STH and their main source of information

\begin{tabular}{|c|c|c|c|c|c|c|c|}
\hline \multirow{2}{*}{$\begin{array}{l}\text { Caregivers' knowledge of } \\
\text { schistosomiasis and STH }\end{array}$} & \multicolumn{7}{|c|}{ Caregivers main source of information on schistosomiasis and STH } \\
\hline & None & Clinic & Research organization & Community meeting & Media & Relatives/friends & $x^{2} p$-value \\
\hline \multicolumn{8}{|l|}{ Schistosomiasis transmission } \\
\hline No idea & $51(89.5)$ & $27(58.7)$ & $38(53,5)$ & $35(38.5)$ & $33(58.9)$ & $60(49.6)$ & \multirow[t]{3}{*}{0.001} \\
\hline Incorrect answers & $0(0.0)$ & $3(6.5)$ & $11(15.5)$ & $15(16.5)$ & $7(12.5)$ & $27(22.3)$ & \\
\hline At least one correct answer & $6(10.5)$ & $16(34.8)$ & $22(31.0)$ & $41(45.1)$ & $16(28.6)$ & $34(28.1)$ & \\
\hline \multicolumn{8}{|l|}{ Schistosomiasis prevention } \\
\hline No idea & $49(86.0)$ & $7(37.0)$ & $26(36.6)$ & $33(36.3)$ & $8(32.1)$ & $49(40.5)$ & \multirow[t]{3}{*}{0.001} \\
\hline Incorrect answers & $1(1.8)$ & $4(8.5)$ & $8(11.3)$ & $10(11.0)$ & $15(26.8)$ & $21(17.4)$ & \\
\hline At least one correct answer & $7(12.3)$ & $25(54.3)$ & $37(52.1)$ & $48(52.7)$ & $23(42.1)$ & $51(42.1)$ & \\
\hline \multicolumn{8}{|c|}{ Schistosomiasis signs and symptoms } \\
\hline No idea & $57(100.0)$ & $12(26.1)$ & $24(33.8)$ & $31(43.1$ & $5(26.8)$ & 37 (30.6) & \multirow[t]{3}{*}{0.001} \\
\hline Incorrect answers & $0(0.0)$ & $1(2.2)$ & $3(4.2)$ & $22(24.2)$ & $9(16.1)$ & $27(22.3)$ & \\
\hline At least one correct answer & $0(0.0)$ & $33(71.7)$ & $44(62.0)$ & $38(41.8)$ & $32(57.1)$ & $57(57,1)$ & \\
\hline \multicolumn{8}{|l|}{ STH transmission } \\
\hline No idea & $28(49.1)$ & $27(58.7)$ & $43(60.6)$ & $38(41.8)$ & $31(55.4)$ & $74(61.2)$ & \multirow[t]{3}{*}{0.157} \\
\hline Incorrect answers & $9(15.8)$ & $5(10.9)$ & $11(15.5)$ & $16(17.6)$ & $11(19.6)$ & $21(17.4)$ & \\
\hline At least one correct answer & $20(35.1)$ & $14(30.4)$ & $17(23.9)$ & $37(40.7)$ & $14(25.0)$ & $26(21.5)$ & \\
\hline \multicolumn{8}{|l|}{ STH prevention } \\
\hline No idea & $14(24.6)$ & $22(47.8)$ & $30(42.3)$ & $28(30.8)$ & $21(37.5)$ & $43(35.5)$ & \multirow[t]{3}{*}{0.040} \\
\hline Incorrect answers & $11(19.3)$ & $7(15.2)$ & $10(14.1)$ & $21(23.1)$ & $12(21.4)$ & $38(31.4)$ & \\
\hline At least one correct answer & $32(56.1)$ & $17(37.0)$ & $31(43.7)$ & $42(46.2)$ & $23(41.1)$ & $40(33.1)$ & \\
\hline \multicolumn{8}{|l|}{ STH signs and symptoms } \\
\hline No idea & 22 (38.6) & $21(45.7)$ & $37(52.1)$ & $46(50.5)$ & $29(51.8)$ & $52(43.0)$ & \multirow[t]{3}{*}{0.720} \\
\hline Incorrect answers & $5(8.8)$ & $5(10.9)$ & $8(11.3)$ & $10(11.0)$ & $8(14.3)$ & $12(9.9)$ & \\
\hline At least one correct answer & $30(52.6)$ & $20(43.5)$ & $26(36.6)$ & $35(38.5)$ & $19(33.9)$ & $57(47.1$ & \\
\hline
\end{tabular}




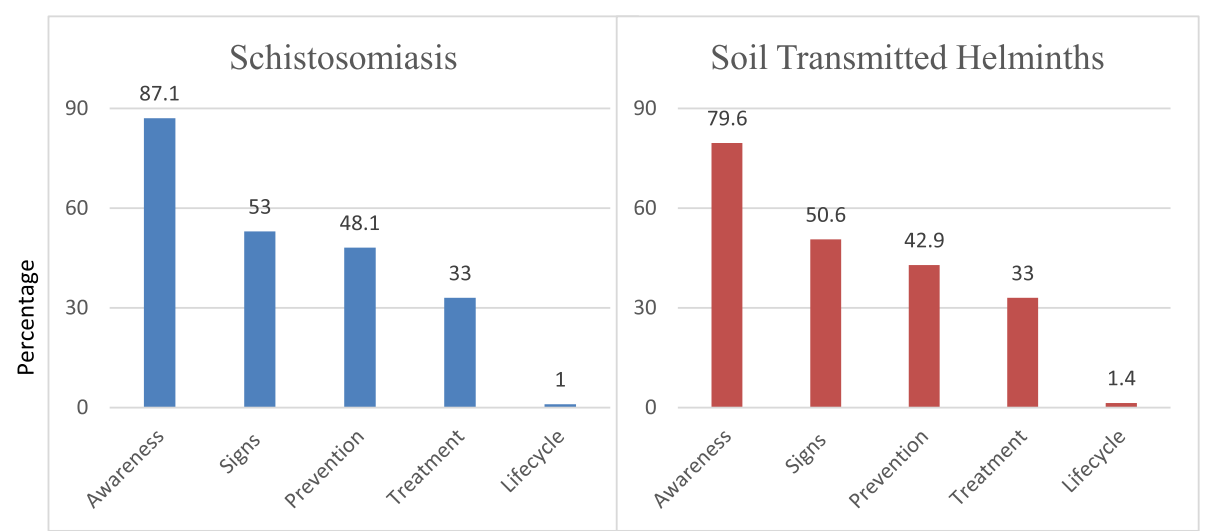

Fig. 2 Schistosomiasis and STH awareness, knowledge of signs, symptoms, transmission, life cycle and prevention among caregivers

knowledge gaps arose from study participants who declared not having any information on schistosomiasis ranging from $30.9 \%$ on signs and symptoms and $95.1 \%$ on the life cycle. Consequently, only about half of the sub-sample that had heard of schistosomiasis could accurately name a specific symptom (53\%), knew about prevention $(48.1 \%)$ while $33 \%$ had knowledge on treatment and control and 1\% knew could describe the life cycle. Overall, the proportion of sampled participants with knowledge of schistosomiasis ranged from $0.23 \%$ on the life cycle to $42.15 \%$ on signs and symptoms.

\section{STH awareness, knowledge of signs, symptoms,} transmission, life cycle and prevention among caregivers Figure 3 presents overall findings on knowledge of soiltransmitted helminths in the Ingwavuma area of uMkhanyakude. Although $79.6 \%$ of participants had heard about $\mathrm{STH}$, a large proportion did not have detailed knowledge. Almost two thirds (62.2\%) of participants knew some signs and symptoms of STH. However, only $2.3 \%$ of participants confirmed knowledge of STH life cycle. Effective knowledge was poor, lower proportions of the sample correctly listed at least a single STH sign/ symptom $(50.6 \%)$, cause $(32.4 \%)$, life cycle $(1.4 \%)$ or prevention measure $(42.9 \%)$.

\section{Caregivers' knowledge of schistosomiasis and STH signs, symptoms, life cycle, transmission and prevention} Results show significant misconceptions on signs and symptoms, transmission, life cycle and prevention of schistosomiasis (Table 2). Participants related sexual practices and symptoms of sexually transmitted diseases with schistosomiasis citing genital sores as being symptomatic of schistosomiasis, unprotected intercourse as a transmission mode and protected intercourse as a preventive measure. Other notable misconceptions

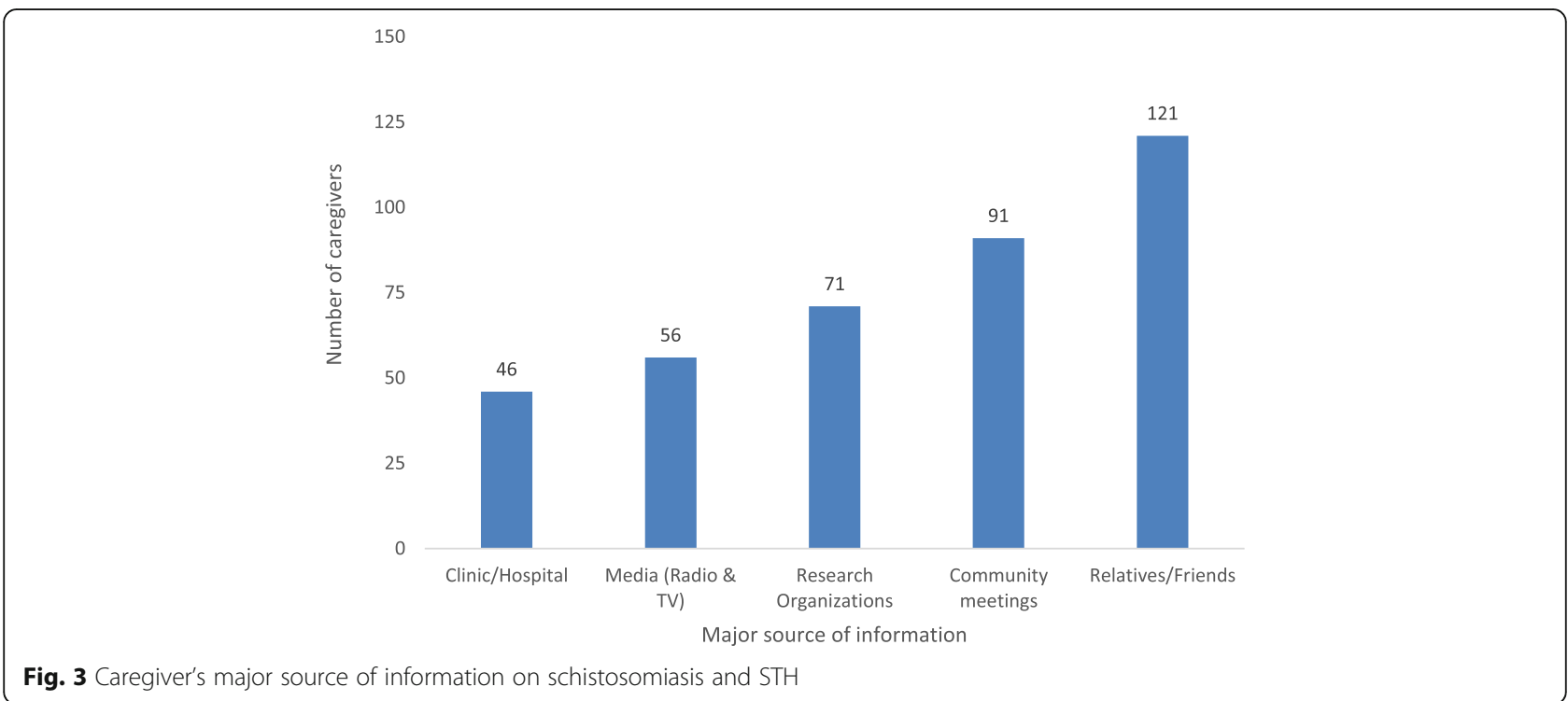


Table 2 Association between caregiver's knowledge of schistosomiasis and STH and attitudes towards these infections

\begin{tabular}{|c|c|c|c|c|}
\hline \multirow{2}{*}{$\begin{array}{l}\text { Caregivers' attitudes towards } \\
\text { schistosomiasis }\end{array}$} & \multicolumn{4}{|c|}{ Caregivers' knowledge about schistosomiasis prevention and control } \\
\hline & No idea & Incorrect answers & At least one correct & $x^{2} p$-value \\
\hline \multicolumn{5}{|c|}{ Schistosomiasis is a minor infection that may clear overtime without treatment } \\
\hline Strongly agree & $97(51.3)$ & $30(15.9)$ & $62(32.8)$ & \multirow[t]{4}{*}{0.001} \\
\hline Somewhat agree & $43(50.0)$ & $10(11.6)$ & $33(38.4)$ & \\
\hline Somewhat disagree & $25(36.2)$ & $9(13.0)$ & $35(50.7)$ & \\
\hline Strongly disagree & $27(27.6)$ & $10(10.2)$ & $61(62.2)$ & \\
\hline \multicolumn{5}{|c|}{ Whether schistosomiasis treatment results in severe, long-term side effects } \\
\hline Strongly agree & $107(46.9)$ & $29(12.7)$ & $92(40.4)$ & \multirow[t]{4}{*}{0.572} \\
\hline Somewhat agree & $31(39.7)$ & $13(16.7)$ & $34(43.6)$ & \\
\hline Somewhat disagree & $24(46.2)$ & $5(9.6)$ & $23(44.2)$ & \\
\hline Strongly disagree & $30(35.7)$ & $12(14.3)$ & $42(50.0)$ & \\
\hline \multicolumn{5}{|l|}{ Schistosomiasis treatment is costly } \\
\hline Strongly agree & $68(47.9)$ & $16(11.3)$ & $58(40.8)$ & \multirow[t]{5}{*}{0.015} \\
\hline Somewhat agree & $36(47.4)$ & $16(21.1)$ & $24(31.6)$ & \\
\hline Somewhat disagree & $43(46.7)$ & $13(14.1)$ & $36(39.1)$ & \\
\hline Strongly disagree & $45(34.1)$ & $14(10.6)$ & $73(55.3)$ & \\
\hline Caregivers' attitudes towards STH & Caregivers' & of STH prevention a & & \\
\hline \multicolumn{5}{|c|}{ STH are minor infections that cause no harm to the body } \\
\hline Strongly agree & $14(24.1)$ & $16(27.6)$ & $28(48.3)$ & \multirow[t]{4}{*}{0.018} \\
\hline Somewhat agree & $27(48.2)$ & $12(21.4)$ & $17(30.4)$ & \\
\hline Somewhat disagree & $29(44.6)$ & $18(27.7)$ & $18(27.7)$ & \\
\hline Strongly disagree & $88(33.5)$ & $53(20.2)$ & $122(46.4)$ & \\
\hline \multicolumn{5}{|l|}{ STH only affects adults and not children } \\
\hline Strongly agree & $32(35.2)$ & $21(23.1)$ & $38(41.8)$ & \multirow[t]{4}{*}{0.203} \\
\hline Somewhat agree & $36(45.0)$ & $18(22.5)$ & $26(32.5)$ & \\
\hline Somewhat disagree & $31(39.2)$ & $20(25.3)$ & $28(35.4)$ & \\
\hline Strongly disagree & $59(30.7)$ & $40(20.8)$ & $93(48.4)$ & \\
\hline \multicolumn{5}{|l|}{ STH treatment is costly } \\
\hline Strongly agree & $37(43.0)$ & $22(25.6)$ & $27(31.4)$ & \multirow[t]{4}{*}{0.021} \\
\hline Somewhat agree & $38(45.2)$ & $20(23.8)$ & $26(31.0)$ & \\
\hline Somewhat disagree & $25(29.8)$ & $21(25.0)$ & $38(45.2)$ & \\
\hline Strongly disagree & $58(30.9)$ & $36(19.1)$ & $94(50.0)$ & \\
\hline
\end{tabular}

included transmission of schistosomiasis through feeding practices and including the soil as part of the schistosomiasis life cycle. Misconceptions were also widespread on signs and symptoms, causes, life cycle and prevention of STH. The misconceptions included teeth grinding, dietary habits and treatment as signs, transmission ways and preventive methods respectively.

\section{Caregivers' knowledge of schistosomiasis and STH and their main source of information}

Out of 385 caregivers who acknowledged to have heard about schistosomiasis and STH, friends/relatives $121(31.4 \%)$ and community meetings $91(23.6 \%)$ were the most reported as major sources of information while clinics/hospitals were the least reported 46(11.9) source (see Fig. 3).

Caregivers' knowledge of schistosomiasis and STH and their main source of information Table 3 shows that caregiver's knowledge on the transmission was associated with the source of information ( $p$-value: 0.001$)$ and most participants with correct responses received the information in community meetings. The caregivers' knowledge of schistosomiasis prevention, signs and symptoms were also associated with the main source of information (p-value: 0.001). Those knowledgeable received the information from community meetings and 


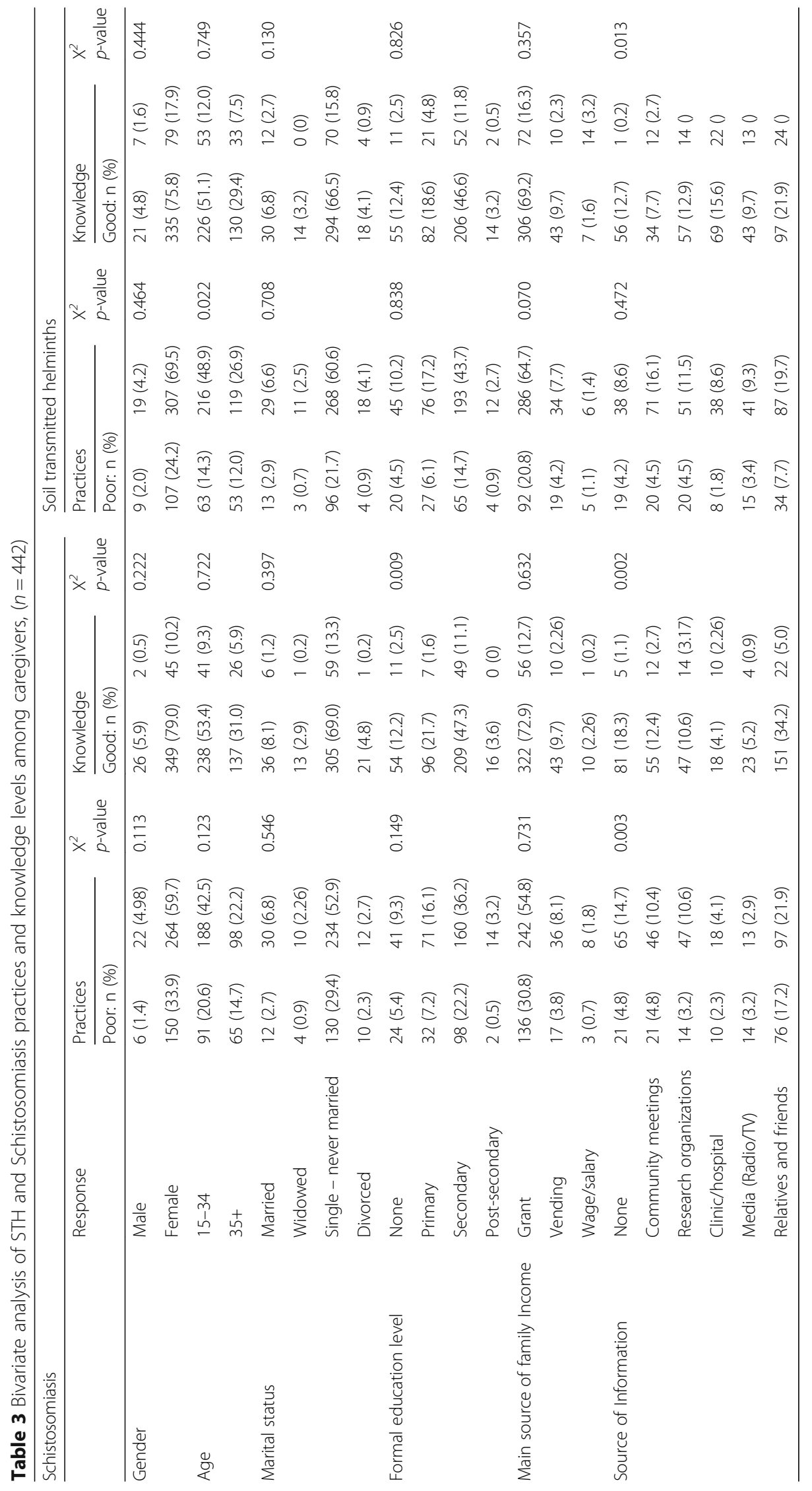


relatives-friends. For STH, only the knowledge on prevention was associated with the source of information and most of the caregivers were also informed through community meetings.

\section{Knowledge, attitudes, practices and control of schistosomiasis and STH}

Five themes emerged from the data: (i) preferred sources of information, (ii) knowledge and misconception, (iii) attitudes (iv) water and sanitary practices (v) control and (vi) health-seeking behaviour (see Table 4). Most of the study participants reported to have inadequate knowledge, attitudes on modes of transmission, life cycle, prevention and control "As for me, I don't have good information about these diseases, but I remember growing up, our elders used to warn us against jumping over fire and even eating cold food"; Male respondent - FGD. Noted misconceptions were related to the schistosomiasis and STH transmission, signs and symptoms, and prevention. FGD participants mentioned that schistosomiasis may be caused by sexual intercourse, jumping over fire, and drinking dirty water.

Most participants, however, were able to mention at least one sign or symptom of schistosomiasis and the most mentioned was "blood in urine". Most participants preferred visiting clinics/hospitals as opposed to traditional healers. However, they mentioned that if they do not get better when using modern medicine, they resort to traditional healers or sometimes use both medications at the same time. Noted misconceptions were mostly related to the schistosomiasis and STH transmission and prevention. FGD participants mentioned that Schistosomiasis may be caused by sexual intercourse, jumping over fire, and drinking dirty water.

\section{Association between caregiver's knowledge on schistosomiasis and STH and attitudes towards these infections}

Additional file 1: Table S1 shows the association between caregiver's knowledge and attitudes towards schistosomiasis and STH; Respondents who regarded schistosomiasis and STH as minor infections that could heal without any intervention ( $p$-value: 0.001$)$ and assumed that schistosomiasis treatment was costly $(p$ value: 0.001 ) were more likely to have a negative attitude towards schistosomiasis control and prevention. The same trend was also observed with STH.

\section{Bivariate analysis of STH and schistosomiasis practices and knowledge levels among caregivers in Ingwavuma} Results from the bivariate analysis showed a significant association between age group and source of family income and practices on both STH and schistosomiasis (see Additional file 2: Table S2 and Additional file 3: Table S3). Formal education attainment and source of information was significantly associated with knowledge

Table 4 Binary logistic regression analysis of STH and Schistosomiasis practices and knowledge levels among caregivers in Ingwavuma

\begin{tabular}{|c|c|c|c|c|c|}
\hline \multirow[t]{2}{*}{ Disease } & \multirow[t]{2}{*}{ Variable } & \multirow[t]{2}{*}{ Categories } & \multirow{2}{*}{$\begin{array}{l}\text { Practices } \\
\text { COR }(95 \% \mathrm{Cl})\end{array}$} & \multicolumn{2}{|l|}{ Knowledge Level } \\
\hline & & & & COR $(95 \% \mathrm{Cl})$ & AOR $(95 \% \mathrm{Cl})$ \\
\hline \multirow[t]{9}{*}{ STH } & \multirow[t]{3}{*}{ Formal education level } & No formal schooling & - & 1 & 1 \\
\hline & & Primary & - & $0.935(0.456-1.918)$ & $1.010(0.481-2.122)$ \\
\hline & & Secondary and above & - & $0.335(0.146-0.766)^{a}$ & $0.344(0.148-0.798)^{a}$ \\
\hline & \multirow[t]{6}{*}{ Source of Information on STH } & None & 1 & 1 & 1 \\
\hline & & Community meetings & $2.425(1.363-4.316)^{a}$ & $0.424(0.155-1.161)$ & $0.447(0.162-1.233)$ \\
\hline & & Research organizations & $1.716(0.945-3.118)$ & $1.498(0.695-3.228)$ & $1.632(0.749-3.555)$ \\
\hline & & Clinic/hospital & $2.630(1.349-5.130)^{\mathrm{a}}$ & $2.044(0.970-4.310)$ & 2.109 (0.993-4.482) \\
\hline & & Media (Radio/TV) & $1.410(0.615-3.232)$ & $3.813(1.561-9.314)^{a}$ & $3.744(1.518-9.232)^{a}$ \\
\hline & & Relatives and friends & $0.728(0.323-1.639)$ & $1.194(0.377-3.778)$ & $1.453(0.450-4.698)$ \\
\hline \multirow[t]{8}{*}{ Schistosomiasis } & \multirow[t]{2}{*}{ Age group } & $15-34$ & 1 & - & - \\
\hline & & $35-70$ & $1.652(1.073-2.543)^{a}$ & - & - \\
\hline & \multirow[t]{6}{*}{ Source of Information on STH } & None & & 1 & - \\
\hline & & Community meetings & & $0.072(0.010-0.548)^{a}$ & - \\
\hline & & Research organizations & & $1.426(0.644-3.160)$ & - \\
\hline & & Clinic/hospital & & $0.993(0.476-2.072)$ & - \\
\hline & & Media (Radio/TV) & & $1.289(0.669-2.483)$ & - \\
\hline & & Relatives and friends & & $1.222(0.569-2.625)$ & - \\
\hline
\end{tabular}


of STH while the source of health information was the only significant variable for schistosomiasis knowledge.

\section{Binary logistic regression of STH and schistosomiasis practices and knowledge levels among caregivers in Ingwavuma}

From the multivariate analysis, caregivers who got information through community meetings (COR 2.425, 95\% CI: 1.363-4.316) and health institutions (COR 2.630, 95\% CI: 1.349-5.130) were more likely to report good STH practices than those reporting not receiving any information (see Table 4). Caregivers with at least secondary education were $66 \%$ (COR 0.335 , 95\% CI: $0.146-$ 0.766) less likely to have high knowledge of STH relative to their counterparts without any formal education. On the other hand, caregivers who accessed health information through the media had higher odds (COR 3.813, 95\% CI: 1.561-9.314) of attaining high knowledge levels on STH when compared with caregivers not receiving any information. However, there was no noticeable change in the likelihood of attaining higher knowledge levels on STH for caregivers at least secondary education (AOR 3.744, 95\% CI: 1.518-9.232) those accessing information through the media (AOR 0.344, 95\% CI: 0.1480.798) after controlling for other variables. Being aged at least 35 years increased the odds of reporting good practices on schistosomiasis by $65 \%$ (COR 1.652, 95\% CI: 1.073-2.543) while receiving health information through community meetings (COR 0.072, 95\% CI: 0.010-0.548) significantly reduced the odds of having good knowledge on schistosomiasis (Additional file 2: Table S2).

\section{Discussion}

Major sources of information on schistosomiasis and STH This study shows a significant relationship between caregiver's major source of information and the ability to give at least one correct answer. Our study revealed that most people relied on family friends and community meetings for health information. Caregivers that could not give accurate responses on schistosomiasis and STH were those that relied on family and friends for schistosomiasis and STH related information. Our findings are in line with previous studies that found that peers and family members tend to give inaccurate health messages $[29,30]$ due to limited health-related knowledge [31]. This suggests therefore that, if community members are empowered with accurate information, schistosomiasis and STH control programs can leverage on them as existing dissemination channels, in order to halt the spread of infection through behaviour change strategies.

\section{Knowledge and practices on schistosomiasis and STHs}

This study aimed to understand knowledge, attitudes and practices on schistosomiasis and STH among caregivers in rural KwaZulu-Natal, South Africa. Our study provides invaluable insights into KAP in rural South Africa. There was education intervention among participants prior to the study. The establishment of the level of knowledge and misconception prior to an intervention program was important to design context-specific educational programs on schistosomiasis and STH. Most respondents claimed to be aware of schistosomiasis and STH. however, knowledge levels declined drastically with follow-up questions assessing the accuracy of the self-reported knowledge. Knowledge was highest on signs and symptoms and the least on life cycle for both schistosomiasis and STH. The low knowledge was prevalent across categories of key caregivers' socio-demographic characteristics. The low level of knowledge despite high awareness levels have been reported widely by KAP studies conducted in Bangladesh [32], Kenya [33], South Africa [34] and Ethiopia [35]. A recent systematic review on KAP of schistosomiasis in SSA reported low levels of comprehensive knowledge despite high levels of self-reported disease awareness ranging from between 75 and 98\% [15]. This strongly suggests communities may overestimate their health-related knowledge which may, in turn, hinder the effectiveness of treatment and control programs and has implications for health promotion intervention. For this reason, contextual realities ought to be considered when designing health awareness campaigns particularly in rural areas with low levels of formal education. Risky water and sanitary related practices were observed among most of the participants who reported relying on open water sources due to water scarcity and whose children practice open defecation due to the unavailability of appropriate toilets for PSAC. Previous studies have overemphasized the importance of water, sanitation and hygiene (WASH) in the control of schistosomiasis and STH [36]. Disadvantaged communities, however, are still grappling with the provision of such services due to financial constraints and expertise required for their implementation.

\section{Attitudes and misconceptions on schistosomiasis and STHs infection}

Our findings revealed widespread misconceptions on schistosomiasis and STH among all participants and respondents i.e. community caregivers, traditional leaders, preschool teachers, caregivers in the Ingwavuma community. Most fallacies related to schistosomiasis signs and symptoms, transmission and prevention. For instance, participants in FGDs and in-depth interviews confused schistosomiasis with sexually transmitted infections due to the blood in urine. Our findings are consistent with previous findings from other settings including Tanzania [37], Mozambique [38], and Kenya [39, 40]. On the other hand, the few misconceptions on STH were seemingly mythical. Rural communities are often 
marked by dominant cultural influences characterized by widespread close-knit structures of influence thus wrong messages may easily lead to widespread misconceptions and risky practices that cascade across generations [41]. The distortion of health seeking behaviour resulting from misconceptions and myths on illness may hinder control efforts in the case of schistosomiasis and STH.

Most male participants in our study underestimated the seriousness of schistosomiasis and $\mathrm{STH}$, rating them as infections that not cause death easily. Earlier studies from Uganda [42], Tanzania [37], Kenya [43] and Nigeria [20] had similar findings where respondents believed schistosomiasis was a natural disease that did not require medical attention. In our study, FGDs with men also revealed that they believed it was the responsibility of women to acquire health information because their cultural and ordained role is to take care of their children and the entire family. Contrary to this belief, earlier systematic review findings reported that women had lower levels of health-related knowledge compared to men [15], however, men tend to have poor healthseeking behaviour compared to women [44]. Such variations in beliefs and practices, however, are not surprising considering that in most rural areas in African societies, human behaviour is mainly governed by strong sociocultural influences and expectations [45]. Such beliefs, however, may become bottlenecks for control interventions. Ultimately, they hinder programs from timely controlling and eliminating Schistosomiasis and STH in such settings.

\section{Conclusion}

The low levels of awareness, poor attitudes and practices observed in this study highlight major deficiencies in current strategies to control schistosomiasis and STH. To improve disease control at the community level, it is important to include community stakeholders in developing and delivering awareness programs. This will further enhance the efficiency of communal engagement and information dissemination channels. Developmental interventions as safe water provision are required to minimize exposure to WASH and livelihood activities predispose the community to infection are also required. Finally, it is important to incorporate strategies that address cultural misconceptions and myths on disease into health awareness activities and control measures.

\section{Additional files}

Additional file 1: Table S1. Sociodemographic characteristics of caregivers. Describes the overall sociodemographic characteristics of 442 caregivers of PSAC who were screened for schistosomiasis and STH in uMkhanyakude district. (DOCX $16 \mathrm{~kb}$ )
Additional file 2: Table S2. Caregivers' knowledge on the signs and symptoms, life cycle, transmission and prevention of schistosomiasis and STH. Is a frequency table showing caregivers' knowledge of schistosomiasis and STH signs and symptoms, life cycle, transmission and prevention. (DOCX 16 kb)

Additional file 3: Table S3. Knowledge, attitudes, practices and control of schistosomiasis and STH. Shows FGDs and in-depth interview themes, descriptions and verbatim quotes from CCGs and caregivers. (DOCX $18 \mathrm{~kb}$ )

\section{Abbreviations}

CCG: Community caregiver; CHW: Community health worker; FGD: Focus Group Discussion; KAP: Knowledge, attitudes and practices; PSAC: Preschool age children; SAC: School-aged children; STH: Soil-transmitted helminths; WASH: Water Sanitation and Hygiene; WHO: World Health Organization

\section{Acknowledgements}

The views expressed in this publication are those of the author(s) and not necessarily those of the NHSP, the National Institute of Health Research, or the Department of Health. The authors further acknowledge the KwaZulu-Natal Department of Health, uMkhanyakude district health office, Ingwavuma area community leaders and members who supported the study.

\section{Authors' contributions}

HS and MC developed the concept. HS did the analysis and reporting MC and MK guided the process of analysis, reporting and manuscript writing. All the authors have read and approved the final article.

\section{Funding \\ This research was commissioned by the National Institute of Health Research using Official Development Assistance (ODA) funding. The study was mainly funded by the University of Edinburgh under the British Academy Project. The University of KwaZulu-Natal also supported the study through the College of Health Sciences Scholarship. HS is funded by the Medical Research Council of South Africa through a PhD scholarship under the National Health Scholars Program (NHSP). The funders had no role in designing this study, preparation of the manuscript and decision to have it published. Furthermore, the views, opinions, assumptions or any other information presented in this manuscript are solely those of the authors.}

\section{Availability of data and materials}

The datasets used and/or analysed during the current study are available from the corresponding author on reasonable request.

\section{Ethics approval and consent to participate}

The study was conducted after obtaining ethical approvals from the University of KwaZulu-Natal Biomedical Research Ethics Committee (BREC), Department of Health (DoH) and Provincial Health and Research Ethics Committee (PHREC); and the Department of Social Development in South Africa, KwaZulu-Natal province. Gatekeeper permission was also granted by uMkhanyakude district community leaders, primary schools, ECD centres and clinic facilities. Caregivers who participated in the study (parents and guardians) willingly consented and they also signed written consent forms on behalf of their children. The study gathered evidence useful to programs and organization involved in the prevention and control of schistosomiasis and STH.

\section{Consent for publication}

Not applicable.

\section{Competing interests}

The authors declare that they have no competing interests. 
Received: 15 January 2019 Accepted: 3 July 2019

Published online: 22 August 2019

\section{References}

1. Hotez $\mathrm{P}$, et al. Recent progress in integrated neglected tropical disease control. Trends Parasitol. 2007;23(11):511-4.

2. Brooker S, Clements AC, Bundy DA. Global epidemiology, ecology and control of soil-transmitted helminth infections. Adv Parasitol. 2006;62:221-61.

3. Richardson ST, et al. Global health: urogenital schistosomiasis in the adolescent girl. J Pediatr Adolesc Gynecol. 2016;29(4):326-32.

4. Pullan RL, et al. Global numbers of infection and disease burden of soil transmitted helminth infections in 2010. Parasit Vectors. 2014;7(1):37.

5. Salim N, et al. Distribution and risk factors for Plasmodium and helminth coinfections: a cross-sectional survey among children in Bagamoyo district, coastal region of Tanzania. PLoS Negl Trop Dis. 2015;9(4):e0003660.

6. Schmidlin T, et al. Effects of hygiene and defecation behavior on helminths and intestinal protozoa infections in Taabo, Côte d'Ivoire. PLoS One. 2013;8(6):e65722.

7. Bundy DA, et al. 100 years of mass deworming programmes: a policy perspective from the World Bank's disease control priorities analyses. In: Advances in parasitology. Amsterdam: Elsevier; 2018. p. 127-54.

8. Campbell SJ, et al. Tailoring water, sanitation, and hygiene (WASH) targets for soil-transmitted helminthiasis and schistosomiasis control. Trends Parasitol. 2018;34(1):53-63.

9. Raso G, et al. An integrated approach to control soil-transmitted helminthiasis, schistosomiasis, intestinal Protozoa infection, and diarrhea: protocol for a cluster randomized trial. JMIR Res Protoc. 2018;7(6):e145

10. Clarke NE, et al. (S) WASH-D for Worms: a pilot study investigating the differential impact of school-versus community-based integrated control programs for soil-transmitted helminths. PLoS Negl Trop Dis. 2018;12(5): e0006389.

11. Pabalan $\mathrm{N}$, et al. Soil-transmitted helminth infection, loss of education and cognitive impairment in school-aged children: a systematic review and meta-analysis. PLoS Negl Trop Dis. 2018;12(1):e0005523.

12. Salam, R.A., et al., Mass deworming for soil-transmitted helminths and schistosomiasis among pregnant women: a systematic review and individual participant data meta-analysis. 2018

13. Bronzan RN, et al. Impact of community-based integrated mass drug administration on schistosomiasis and soil-transmitted helminth prevalence in Togo. PLoS Negl Trop Dis. 2018;12(8):e0006551.

14. Schatz EJ. "Taking care of my own blood": older women's relationships to their households in rural South Africa. Scand J Public Health Suppl. 2007;69:147-54.

15. Sacolo H, Chimbari M, Kalinda C. Knowledge, attitudes and practices on schistosomiasis in sub-Saharan Africa: a systematic review. BMC Infect Dis. 2018;18(1):46

16. Moodley I, et al. Temperature-suitability maps for schistosomiasis in South Africa. Ann Trop Med Parasitol. 2003;97(6):617-27.

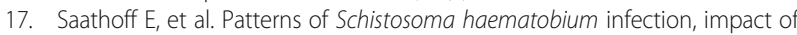
praziquantel treatment and re-infection after treatment in a cohort of schoolchildren from rural KwaZulu-Natal/South Africa. BMC Infect Dis. 2004; 4(1):40.

18. Randjelovic A, et al. A study of hurdles in mass treatment of schistosomiasis in KwaZulu-Natal, South Africa. S Afr Fam Pract. 2015;57(2):57-61.

19. Kihara J, et al. Drug efficacy of praziquantel and albendazole in school children in Mwea, Central Province, Kenya. Acta Trop. 2007;102:165-71

20. Ekpo UF, et al. Urinary schistosomiasis among preschool children in a rural community near Abeokuta, Nigeria. Parasit Vectors. 2010;3:58.

21. Southern, H.S., Africa (Pty) Ltd Institute of Natural Resources. 2003.

22. Kabuyaya $\mathrm{M}$, et al. Schistosomiasis risk factors based on the infection status among school-going children in the Ndumo area, uMkhanyakude district, South Africa. South Afr J Infect Dis. 2017;32(2):67-72.

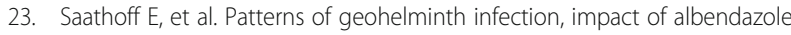
treatment and re-infection after treatment in schoolchildren from rural KwaZulu-Natal/South-Africa. BMC Infect Dis. 2004;4:27.

24. Cochran WG. Sampling techniques: Wiley; 2007.

25. Kabuyaya $M$, et al. Efficacy of praziquantel on Schistosoma haematobium and re-infection rates among school-going children in the Ndumo area of uMkhanyakude district, KwaZulu-Natal, South Africa. Infect Dis Poverty. 2017; 6(1):83.
26. Kabuyaya M, Chimbari MJ, Mukaratirwa S. Infection status and risk factors associated with urinary schistosomiasis among school-going children in the Ndumo area of uMkhanyakude district in KwaZulu-Natal, South Africa two years post-treatment. Int J Infect Dis. 2018;71:100-6.

27. Khan ME, et al. The use of focus groups in social and behavioural research: some methodological issues. World Health Stat Q. 1991;44(3):145-9.

28. Deniau, C., et al., Using the KoBoCollect tool to analyze the socio-economic and socio-cultural aspects of commercial hunting and consumption of migratory waterbirds in the lakes Chad and Fitri (Chad). 2017.

29. Gyorkos TW, et al. Impact of health education on soil-transmitted helminth infections in schoolchildren of the Peruvian Amazon: a cluster-randomized controlled trial. PLoS Negl Trop Dis. 2013;7(9):e2397.

30. Olsen A. Experience with school-based interventions against soil-transmitted helminths and extension of coverage to non-enrolled children. Acta Trop. 2003;86(2-3):255-66.

31. Monse $B$, et al. The fit for school health outcome study-a longitudinal survey to assess health impacts of an integrated school health programme in the Philippines. BMC Public Health. 2013;13(1):256.

32. Nath $\mathrm{TC}$, et al. Elimination of soil-transmitted helminthiasis infection in Bangladesh: knowledge, attitudes, and practices regarding mass drug administration. J Glob Health Rep. 2018;2.

33. Masaku J, et al. High prevalence of helminths infection and associated risk factors among adults living in a rural setting, Central Kenya: a crosssectional study. Trop Med Health. 2017:45(1):15.

34. Sibiya JE, Gumbo JR. Knowledge, attitude and practices (KAP) survey on water, sanitation and hygiene in selected schools in Vhembe District, Limpopo, South Africa. Int J Environ Res Public Health. 2013;10(6):2282-95.

35. Vivas A, et al. Knowledge, attitudes, and practices (KAP) of hygiene among school children in Angolela, Ethiopia. J Prev Med Hyg. 2010;51(2):73.

36. Campbell SJ, et al. Water, sanitation and hygiene (WASH) and environmental risk factors for soil-transmitted helminth intensity of infection in Timor-Leste, using real time PCR. PLoS Negl Trop Dis. 2017;11(3):e0005393.

37. Onyeneho NG, et al. Perceptions, attitudes and practices on schistosomiasis in Delta State, Nigeria. Tanzan J Health Res. 2010;12(4):287-98.

38. Rassi C, et al. Have you heard of schistosomiasis? Knowledge, attitudes and practices in Nampula Province, Mozambique. PLoS Negl Trop Dis. 2016;10(3):e0004504.

39. Mwai J, Njenga S, Barasa M. Knowledge, attitude and practices in relation to prevention and control of schistosomiasis infection in Mwea Kirinyaga county, Kenya. BMC Public Health. 2016;16(1):819.

40. Alaii, J., et al., Community health workers' experiences and perspectives on mass drug administration for schistosomiasis control in western Kenya. 2012.

41. Koffi AJdA, et al. Community knowledge, attitudes and practices related to schistosomiasis and associated healthcare-seeking behaviours in northern Côte d'Ivoire and southern Mauritania. Infect Dis Poverty. 2018;7(1):70.

42. Anguza J, et al. Community perception of intestinal schistosomiasis in Busia district of Uganda. East Afr Med J. 2007;84(2):56-66.

43. Omedo $\mathrm{M}$, et al. The effect of a health communication campaign on compliance with mass drug administration for schistosomiasis control in western Kenya--the SCORE project. Am J Trop Med Hyg. 2014;91(5):982-8.

44. Galdas PM, Cheater F, Marshall P. Men and health help-seeking behaviour: literature review. J Adv Nurs. 2005;49(6):616-23.

45. Hines M. Sex-related variation in human behavior and the brain. Trends Cogn Sci. 2010;14(10):448-56.

\section{Publisher's Note}

Springer Nature remains neutral with regard to jurisdictional claims in published maps and institutional affiliations. 